kaum ausschalten können, zumal jene humanen Züge der

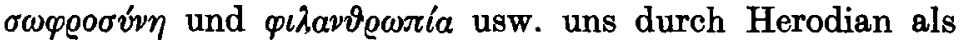
solche des Kaisers selbsit bezeugt werden. Jedenfalls scheint mir unser Edikt ein historisches Dokument von großem Wert zu sein, das noch genauerer Untersuchungen, auch von juristischer Seite, wert ist.

\title{
IX. \\ Das Promptuarium juris des Reichskanzlers und Bischofs Ulrich von Albeck.
}

Ein Beitrag zur Geschichte der populăren Literatur in Österreich.

Von

Herrn Professor Dr. Ivo Pfaff

in Graz.

I.

Eine Pergamenthandschrift der Grazer Universitätsbibliothek Manuskript fol. IV Nr. 23 (alte Nr. 39/2, alte Seckauer Bibl. Nr. 86) enthält ein Promptuarium juris ${ }^{1}$ ) des Ulricus de Albeck. Die Handschrift umfaßt zwei Bände, deren erster 475, deren zweiter 469 Blätter enthält. Die mit alter Paginierung versehenen Blätter sind durch Holzeinbände, welche mit schwarzgefärbtem Leder überzogen sind, zusammengefaßt. Auf den Einbänden befinden sich einfache ornumentale Verzierungen, auf dem Rücken in Gold (stark verblaßt). Die Einbände stammen zweifellos aus dem Stifte Seckau und besitzt die Grazer Bibliothek

1) ther die Wortbedeutung von Promptuarium siehe Forcellini. Lexikon IV 921; Du Cange, Glosearium V; Diefenbach, Glossarium p. 308; zur Geschichte der Vokabularien und verwandter Rechtsenzyklopädien des Mittelalters s. Seckel, Beiträge zur Gesch. beider Rechte I, insbesondere S. $472 \mathrm{ff}$. 
noch eine Reihe ähnlicher Einbände gleicher Provenienz. Die einzelnen Blätter haben eine Höhe von $46,5 \mathrm{~cm}$, eine Breite von $33 \mathrm{~cm}$; jedes Blatt hat zwei beschriebene Spalten; die Höhe derselben ist $\mathbf{3 4 - 3 5} \mathrm{cm}$, die Breite 9,8. Das Linienschema ist gut erhalten. Die Handschrift ist schön geschrieben; doch sind sehr weitgehende Kürzungen verwendet. Auf dem oberen Rande der Blätter findet sich vereinzelt der Beginn eines neuen Artikels durch ein entsprechendes Schlagwort angegeben. An den Rändern der Blätter sind Buchstaben und gelegentliche Vermerke, die der Handsehrift selbst angehören, welche zur Herstellung der Ubersicht dienen sollen. Die einzelnen Abschnitte sind durch rote Kapitelzeichen gekennzeichnet, wie auch die einzelnen Artikel im Text durch rote Schrift markiert werden. Die Schrift ist die gotische Minuskel der ersten Hälfte des 15. Jahrhunderts. Im Texte sind gelegentlich größere verzierte Initialen mit anschließendem Rankenwerk; auch finden sich viele kleine Initialen (rot und blau) mit einfachen Verzierungen in Federzeichnung.

Blatt 1 recto enthält ein großes Titelbild: Christus als Weltenrichter, zu beiden Seiten desselben die geistlichen und weltlichen Stände; der Papst mit den Kardinälen auf der einen, der Kaiser mit seinen Großen, welche die Reichsinsignien (Schwert, Reichsapfel und Zepter) tragen, auf der anderen Seite; zu Füßen Christi kniet der Verfasser, der sein Werk überreicht. Auf diesem ersten Blatte sind auch noch das Wappen von Seckau (segnende Hand) und das Wappen des Verfassers (linke Seite eines springenden Einhorns) sowie Zierranken und einige Blumen angébracht. Das Wappen des Bistums Verden ist es nicht, denn dasselbe ist nach dem alten Siebenacher I Tafel 10 in blau ein weiß und rot geschachteter Rechtbalken.

Die Miniaturen des Werkes sind in der durch Salzburg beeinflußten Art und Weise gefertigt. Die Handschrift wurde, wie die Vorrede besagt, in Seckau geschrieben. Einzelne nachträgliche Zusätze finden sich auf den verschiedensten Blättern; doch sind sie sichtlich von derselben Hand beigefügt; wahrscheinlich ließ sich der Verfasser das Manuskript von Zeit zu Zeit vorlegen und ordnete, bei Durch- 
sicht des bisher Geschriebenen, diese Zusätze an. (Solche finden wir I 43, 118 recto, 120 verso, 121 verso, 130 verso, 163 verso, 188 recto, 198 verso u. a.m.) Gelegentliche scherzhafte Zeichnungen der Abschreiber sowie sonderbare Gestaltungen einzelner Buchstaben, so I 8, 9, 10, 65, 313; II 27 u. a. m. seien gleichfalls erwähnt.

Über die Abfassung des Werkes äußert sich der Verfasser in der Vorrede zum 1. Bande, welche vom Jahre 1422 datiert ist - die Arbeit Albecks muß also im wesentlichen bereits 1422 abgeschlossen gewesen sein -: Anno domini 1408, dum adhuc ecclesiae praeessem Verdensi in provincia Moguntina, hunc laborem in dei nomine ... . subivi. Der erste Band ist per manus Marci Kalichstain Pruteni de Osterrad, Pomeranensis dyecös. geschrieben und am 20.April 1429 vollendet worden; der 2. Band wurde am 30. August desselben Jahres beendet und der Schreiber desselben äußert sich folgendermaßen : Scripta est haec secunda pars promptuarii per Georium Salsatoris de Glatz, plebanum in Kirchpach, capellanum et familiarem praefati reverentissimi patris et dom. dom. Ulrici eps. Sec. decretorum doctoris, finita anno domini 1429 feria tertia, die sanctorum Felicis et Adaucti Martyr. Uber die beiden Schreiber des Promptuariums vermag ich nichts Näheres anzugeben. In den Xenia Bernadina, welche Bd. II, III und IV eine Reihe von Codexschreibern des 15. Jahrhunderts erwähnen, die auf österreichischem Gebiete tätig waren, fehlen ihre Namen. Albeck hat sie wohl aus dem Norden mitgenommen oder sich kommen lassen.

Um nebst den Quellen, deren Inhalt in Schlagwörtern nach dem Alphabet geordnet dargestellt werden soll Bd. I umfaßt die Buchstaben A-I, Bd. II K-Z - , auch die Literatur entsprechend zu berücksichtigen, hat der Verfasser, wie er in seiner Vorrede angibt, für die einzelnen Abschnitte der einzelnen Artikel einige Werke besonders berïcksichtigt und exzerpiert. Die einzelnen Artikel erörtern die diesbezügliche Frage nach der Regelung, welche sie im decretum Gratiani, den Dekretalen, dem liber sextus, den Clementinen und in den libri feudorum erfahren, wobei natürlich gelegentlich der eine oder andere Abschnitt ent- 
Das Promptuar. jur. d. Reichskanzlers u. Bisch. Ulr. v. Albeck. 161

fällt, wenn dem Autor dort für die betreffende Materie nichts Wesentliches gesagt zu sein scheint. Auch ist die Praxis der rota Romana herangezogen. Für das decretum benützte der Vf. das Werk des Archidiaconus (Guido de Baysio) Apparatus ad decretum ${ }^{1}$ ), für die Dekretalen die Distinctiones in lib. V Decretalium des Henricus Bohic ${ }^{2}$ ), für den liber sextus die Lectura super sexto des Petrus de Ancharano ${ }^{\text {) }}$, für die Clementinen die Lectura super Clementinis des Franciscus de Zabarellis ${ }^{4}$ ) und für die libri feudorum den Baldus de Perusio.

Soviel ich zu sehen vermochte, zitiert der Vf. am häufigsten den Archidiaconus, und zwar nicht nur in den dem Decretum gewidmeten Abschnitten der einzelnen nach dem Alphabet geordneten Artikel, sondern auch anderwärts und wird Übereinstimmung wie gelegentlicher Gegensatz immer wieder sorgfältig hervorgehoben. Außer den fünf in der Vorrede genannten Autoren zitiert Vf. bald aus erster, bald aus zweiter Hand eine Reihe von Schriftstellern, von denen hier einige angeführt seien, so: Goffredus de $\operatorname{Trano}^{5}$ ), Johannes Andreae, Petrus de Bellapertica ${ }^{6}$ ), Hugoccio, Bartolomaeus Brixiensis ${ }^{7}$ ), Raynaldus, Abbas ${ }^{8}$ ), Jacobus Rade-

1) Über Guido de Baysio s. Schulte, Quellen II S. 186.

2) Über ihn s. Schulte a. a. O. II 266.

3) Vgl. Schulte a.a. O. II S. 278 ff.; s. auch Pastor, Gesch. der Päpste I S. 160; Schulte, Lohrb., 3. Auf. S. 102.

4) S. Schulte a. a. O. I S. 28. Zabarella aus Padua wurde 6. Juni 1411 vom Papst Johann XXII. zum Kardinal ernannt und starb 26. September 1417 zu Padua; s. Co ronelli, Cronologia universalis S. 176. Zabarella nahm auch am Konstanzer Konzil teil und muß dort wohl Albeck kennengelernt haben. Zu seiner Auffassung über die geplanten Veränderungen der Kirchenverfassung s. Pastor, Gesch. der Päpste I S. 145. Vgl. überdies auch Savigny, Gesch. des röm. R. im Mittelalter VI 229, 284 und Gregorovius, Gesch. der Stadt Rom VI 619, 667.

5) Vgl. Schulte, Gesch. der Quellen und Literatur II S. $88 \mathrm{ff}$.

6) Über die Abkürzungen, welche der Name des Petrus de Bellapertica in den Handschriften erfuhr, s. Schrutka, Dogmengeschichte und Dogmatik der Freigebung I S. 84. Utber ihn als französischen Juristen des 14. Jahrhunderts s. Savigny a. a. O. V S. $27 \mathrm{ff}$.

7) S. Schulte a. a. O. II $83 \mathrm{ff}$. Albeck zitiert ihn bald als Brix., bald als Bart. oder Bar. Brix.

8) Úber Abbas antiquus s. Schulte a. a. O. II 130.

11 Vol. 42 
wicz $^{1}$ ), Egidius Fuscarius ${ }^{2}$ ), Tancred ${ }^{3}$ ), Jacobus de Arizone $^{4}$ ), Accursius, Jason de Mayno, Cynus, Bartolus, Azo, Martinus, Joh. Bassianus, Garsias Hispanus ${ }^{5}$ ), Odardus (wohl Olradus) $^{6}$ ) Johannes Calderinus ${ }^{7}$ ), Guil. Durantis, Roffredus $^{8}$ ), Hostiensis ${ }^{9}$ ), Lapus ${ }^{10}$ ), Gerhardus Monachus ${ }^{11}$ ), Innocenz IV., Johannes de Deo ${ }^{12}$ ), Bernardus Parmen$\operatorname{sis}^{13}$ ) u. a. m.

Ulricus gewährt in den größeren Artikeln immer eine Überschau über die verschiedenen unter den doctores vertretenen Meinungen, so daß sein Werk über den damaligen Stand der diversen Streitfragen häufig einen tauglichen Überblick bietet.

Daß die alphabetische Anordnung manche Wiederholungen mit sich bringt, ist dem Vf. selbstredend bekannt: saepe etiam eadem materia . . . . in diversis literis iteratur; allein so fährt er fort: saepissime repetita majore soliditate firmetur et attentiori memoria conservetur. Daß der Autor das Werk als für die verschiedensten $Z$ wecke geeignet erachtete und daß er nicht bloß denen durch sein Werk dienlich sein will, die pro incumbentibus negotiis nicht imstande sind, sich selber aus den Quellen und der Literatur zu orientieren, sondern auch den Anfänger dabei im Auge hatte, zeigt wohl der Satz der Vorrede: nam in hoc opere lac parvulis, panis adultis, ossa fortioribus ministratur. Zur Übersichtlichkeit und um den Leser auf Punkte des Werkes $\mathrm{zu}$ verweisen, an welchen über das gleiche oder ein zusammenhängendes Thema gesprochen wird, sind in den einzelnen Artikeln durch Buchstaben entsprechende Verwei-

1) Jacob Radewitz de Ihenis, Kanonist; 1405-1411 Lehrer in Erfurt, schrieb eine lectura in decretales; s. Schulte a. a. O. II 376; Muther, Zur Gesch, der Rechtswiss. 213.

2) S. Schulte a. a. $O$.

3) Tancred war Lehrer des Bartol. Brixiensis; s. Schulte a. a. O.

4) S. Schulte a. a. O. II 82.

5) Über diesen Schulte a. a. O. II 140.

6) S. Schulte a. a. O. II 232.

7) Schüler des Johannes Andreae; s. Schulte a. a. O. II $247 \mathrm{ff}$.

8) S. Schulte a. a. O. II 75.

9) S. Schulte a. a. O. II 123.

10) S. Schulte a. a. O. II 238.

11) S. Schulte a. a. O. II 314.

12) S. Schulte a. a. O. II 94.

13) S. Schulte a. a. O. II 114. 
Das Promptuar. jur. d. Reichskanzlers u. Bisch. Ulr. จ. Albeck. 163

sungen gegeben, welche durch die am Rande der Blätter enthaltenen Buchstaben verdeutlicht werden sollen. Dies Promptuarium überschreitet allerdings, soweit mir die populäre Literatur überhaupt bekannt geworden ist, das übliche Maß weitaus; denn das zwei Großfoliobände umfassende Werk hat 944 Blätter, also 1888 Seiten, von denen jede zwei Kolumnen mit regelmäßig 72 Zeilen umfaßt. Geboten wird eine Darstellung des kirchlichen Rechtes in enzyklopädischer Form; manche Artikel enthalten allerdings nichts Juristisches, sondern geben bloß an der Hand der verschiedensten Zitate aus der Bibel und anderen Werken religiöse und ethische Leitregeln für das Verhalten.

Daß das Werk im Kloster Seckau oder auch später anderwärts benutzt worden sein muß, zeigen die an verschiedenen Stellen vorkommenden Notata, welche von anderer Hand als der des Schreibers herrühren.

Der Umfang der einzelnen Artikel ist natürlich verschieden. So finden sich Artikel, die nur wenige Zeilen umfassen, neben äußerst umfangreichen. So hat der Artikel absolutio 12 , alienatio 16 , actio 19 , appellatio 41 , beneficium 18 , causa 12 , citatio 14 , clericus 26 , collatio 10 , confessio 26 , consuetudo 22 , dolus 4 , donatio 11 , dominium 13 , emtio 10 , episcopus 34, excommunicatio 69 , judicium, judex, jus 86 , juramentum 80 , matrimonium 28 , monachus 19 , mors 12 , mulier 9 , negligentia 10 , obligatio 11 , ordo 30 , papa 19 , pater 10 , patronus 14 , peccatum 27 , poena 29 , possessio 30 , prelatus 22, praescriptio 24, privilegium 25, procuratio 34, religio 43 , renunciatio 17 , rescriptum 27 , restitutio 20 , sententia 43 , successio 15 , sponsalia 18 , statutum 21 , testamentum 20 , testis 49 , venditio 11 , voluntas 14 , votum 17 , usura 22 Spalten.

Was die selbständige Tätigkeit des Vf. anlangt - die Hauptarbeit lag ja zweifellos in dem sorgfältigen Exzerpieren und Zusammenstellen -, so äußerte er sich in der Vorrede g]eichfalls darüber mit den Worten: etiam aliqua intersero, quae per doctores suis in locis non tanguntur, quod agnoscere poteris per dictionem ultra vel extra et in his et aliis, quae in hoc opere posui (folgt eine durchlöcherte Stelle) ... mitto me correctioni determinationi 
et emendationi sanctae matris ecclesiae, a qua nullatenus declino ac cuiuscunque melius sentieris .... Vf. polemisiert auch gelegentlich mit den von ihm exzerpierten Autoren, so z. B. Blatt 103 verso gegen die auch vom Archidiaconus vertretene Auffassung über die Bedingung „si deo placuit". Auch gibt er seinen Polemiken immer eine persönliche Note, so z. B. im Artikel dolus . . . , ,credo quod Accursius male loquitur in hoc ..... ideo dico ... u. a. m. Vereinzelt findet sich auch bei Artikeln der Zusatz „Ulricus auctor huius", wie z. B. bei contemplatio (Blatt 139 recto), während es an anderen Stellen, so z. B. beim Artikel „defendere" heißt „Ulricus compilator hujus operis".

Am Schlusse des 2. Bandes äußert sich Ulricus nochmals über seine Tätigkeit in folgenden Worten:

Protestor attamen in fine, quemadmodum in principio praemisi, quod in omnibus, quae secundum diversas materias in hoc opere continentes simpliciter et quanto brevius potius, dictorum doctorum quorum revolvi volumina dicta secutus sum et si ultra eos quaedam per me posita reperiuntur, non de meo sed ab aliis pro majore materiarum declaratione sed prout visum mihi fuit, commodavi.

Ut saltem simplices et parvuli, qui propter librorum multitudinem de singulis investigare non possunt in promptuario saltem quod intendunt, voleant invenire. Supplico igitur iterato legentibus ut insufficienter dicta corrigant et si qua superaddere iudicaverint ad intelligentiam pleniorem superaddant, ut id quod per me simplicem aliqualiter inchoatum est, perducatur ipsorum sollertia ad perfectum, quia imperfectum meum viderunt occuli mei. Ad ipsius gloriam et honorem, qui est Alpha et Omega principii et finis omnium bonorum, qui est deus sublimis et gloriosus, veniens et regnans in saecula saeculorum. Amen.

\section{II.}

Albecks Promptuarium erörtert, soweit es sich mit Rechtsfragen befaßt, nebst dem jus feudorum das kanonische Recht. Eine Untersuchung nach der Richtung, was dies W.erk für den kanonistischen Rechţ̦shistoriker enthält, 
Das Promptuar. jur. d. Reichskanzlers u. Bisch. Ulr. v. Albeck. 165

liegt mir selbstverständlich fern und es seien die Fachmänner hiermit auf dasselbe aufmerksam gemacht. Was das römische Recht betrifft, so findet, soviel ich zu sehen vermag, dies an zahllosen Stellen sorgfältige Berücksichtigung; es wird zwar nicht ex professo besprochen, doch werden die justinianischen Quellen häufig allegiert und zeigt die ganze Art der Darstellung eine gründliche Vertrautheit mit dem römischen Recht. Selbstverständlich finden sich häufig Bibelstellen, Stellen aus den kanonischen Quellen und dem römischen Rechte nebeneinander, als Beleg für eine vorgetragene Ansicht, angeführt. Auch zitiert Ulricus mitunter Augustinus de civ. dei und Ricardus de Sancto victore. ${ }^{1}$ ) Wie stark gelegentlich Artikel vom römischen Recht beeinflußt sind, zeigt unter anderen der Artikel usucapio. In dem etwas über drei Spalten umfassenden Aufsatz sind nicht weniger als 34 Zitate aus den Institutionen, den Digesten und dem Codex nebst Verweisungen auf die Glossen zu verschiedenen Digestenstellen herangezogen. Andererseits finden sich zahlreiche Artikel, in denen es nahegelegen wäre, das römische Recht zu berücksichtigen und dies doch unterlassen wurde, so z. B. im Artikel turpitudo, publiciana (allerdings ist hier auf den Artikel praescriptio verwiesen), superficies (wo die Frage bloß nach den libri feudorum erörtert wird), specificatio (wo bis auf wenige Zeilen ausschließlich die Entscheidungen der rota Romana zugrunde liegen) und confiscatio (wo ausschließlich kanonisches und Lehenrecht zur Sprache kommt).

Die Zahl der Artikel, welche im wesentlichen bloß Verweisungen auf andere Abschnitte des Werkes enthalten (wie z. B. der Artikel Vellejanum), ist begreiflicherweise eine bedeutende.

1) Richard von St. Victor gehört zu den Koryphäen der von Wilhelm von Champeaux gestifteten Schule und war Lehrer und Führer der asketisch-mystischen Richtung der philosophierenden Theologen des 12. Jahrhunderts. Er war ein Schotte, Prior von St. Victor und starb im Jahre 1173. S. über ihn Lo ew'e, Der Kampf zwischen dem Realismus und Nominalismus (Abh. der k. böhmischen Ges. der Wissensch. VI. Folge 8. Bd. (1876). 
Die Artikel, welche den Sprachgebrauch der verschiedenen Teile des Corpus juris can. erörtern, sind zwar meist sehr kurz, aber recht zahlreich; so seien hier insbesondere angeführt die Artikel: absque, absurdum, atrox, circa, coram, cum, de, diu, donec, dudum, etiam, et, etsi, ex, extunc, fieri, iam, idem, uno, infra, mox, olim, nisi, none, non obstante, nostrum, par, per, plus, potius, quasi, quis, quovismodo, ubicunque, vel, vix, ultra, usque. (Usque includat quando vel excludat de restitutione in integrum coram alias constituisse sexta distinctione ubi dicitur quod in fine dicti capituli usque tenetur exclusive; aliquando tenetur inclusive, ut patet digestis de senatoribus l. ultima $\S$ fin. [1. 12 \$ 1 D. I, 9.] Sed distingue de dictione a et usque, quod aut huiusmodi dictiones proferunt a lege aut ab homine etc.)

Beachtenswert scheint mir in dieser Hinsicht das sprachliche Interesse Albecks zu sein, das für seine Zeit immerhin nicht zu den alltäglichen Erscheinungen gehört.

Im nachstehenden seien nun aus der Fülle der Artikel einige herausgegriffen, bei welchen von vornherein eine Berücksichtigung der römischen Rechtsquellen wahrscheinlich erscheint; das kanonische Recht und das Lehenrecht bleiben dabei außer Betracht.

Im Artikel condicio wird gleich anfangs der Digestentitel 35 . 1 in toto zitiert; auch werden immer wieder die römischen Namen Titius und Sejus verwendet.

Zitiert werden $1.13 \S 12$ de ritu nupt. -23. $2,1.2 \S 7$ in fine. D. de donat. 39. 5, 1. $14 \mathrm{D}$. de usu et habit. 7. 8, 1.9 D. de hered. inst. $28.5 ; 1.44 \S 6 \mathrm{D}$. de cond. et dem. 35, 1 , 1. 4 C. de jure fisci 10. 1, l. 1 Cod. de metall. 12. 40, 1. 34 C. de usufr. 3. 33, 1. 39 D. de statulib. 40. 7, 1.25 D. de adopt. 1. 7, 1.33 D. de cond. et dem. 35, 1, 1.213 D. de verb. sign. 50. 16.

Im Artikel dolus werden die einschlägigen Fragen im Zusammenhang mit dem römischen Recht behandelt. Man sieht, wie der Autor beziehungsweise die von ihm exzerpierten Autoren sich in diesen Fragen von der Darstellung der Digesten beeinflussen ließen. Natürlich werden aber auch hier eine Reihe außerhalb dieses Digestentitels gelegener 
Das Promptuar. jur. d. Reichskanzlers u. Bisch. Ulr. v. Albeck. 167

Stellen allegiert, wie 1. $75 \S 3$ D. de verb. obl. 45. 1, 1. $25 \mathrm{D}$. de contrah. empt. 19.1, 1. 26 (27) D. de neg. gest. 3. 5, 1. 14 (15) D. eod., l. 2 \$ 7 ad leg. Jul. de adult. 48, 5, 1. 5 D. de his qui notant. 3. $2,1.4 \S 13 \mathrm{D}$. de doli exc. 44 . 4 u. a. $\mathrm{m}$.

Auch die Lehre vom dominium ist stark romanistisch beeinflußt, obwohl die Unterscheidung zwischen dominium directum und dominium utile eine große Rolle spielt (s. insbesondere Blatt 217). An sehr vielen Stellen werden in beträchtlichem Ausmaße die römischen Quellen zur Stütze der aufgestellten Thesen herangezogen, so exempli gratia: 1. $20 \mathrm{D}$. de accus. $48.2,1.46 \S 3 \mathrm{D}$. de jure fisci 49.14 , l. 9 pr. C. de bon. prose. et dam. 9. 48, 1. 11 D. de vi et vi arm. 43. 16, l. 87 pr. D. ad municip. 50. 1, l. 25 pr. D. act. emti 19. 1, 1.18 \$ $1 \mathrm{D}$. de hered. pet. 5. 3, 1. $30 \mathrm{D}$. de pecul. 15. 11, 1. 11 § 4 D. de exc. rei jud. 44. 2, 1. 14 § $2 \mathrm{D}$. de exc. rei jud. 44. 2, l. 181 D. de verb. sig. 50. 16, 1. 2 C. de jud. 3. 1 , 1. $39 \S 1 \mathrm{D}$. de evict. 21.2 u. a. m.

In der Lehre von der donatio wie in dem Artikel dos berücksichtigt der Autor die römischen Quellen und verwendet sie als Argumente für seine Ansichten, so im ersteren Falle insbesondere die 1. 10 D. de jur. 12. 2 und die 1. 27 $\S 3 \mathrm{D}$. de pact. 2.14 , im letzteren die $1.9 \S 1 \mathrm{D}$. de donat. 39. 5, während, was den Begriff der dos anlangt, mit den Worten: dos quid sit et quomodo consultum est muliebribus auf den Digestentitel de fundo dotali verwiesen wird. Begreiflicherweise bringt es hier wie anderwärts die Anordnung des Stoffes nach Schlagworten mit sich, daß in den einzelnen Artikeln nicht eine Gesamtdarstellung der betreffenden Materie geboten wird, sondern auf vieles, was bei einer systematischen Erörterung im Zusammenhange zu besprechen wäre, auf den unter einem anderen Schlagwort gebotenen Artikel verwiesen wird. So in der Lehre von der dos auf die Artikel divortium, uxor, virgo, usucapio u. a. m.

Aus dem ziemlich umfangreichen Abschnitte, der die Lehre von der emtio behandelt und eine Menge von Kasuistik entwickelt, an der das ganze Werk reich ist, seien als Hinweise auf die römischen Quellen erwähnt: $1.1 \S 2$ de aed. ed. 21. 1, l. 1 D. de jur. et facti ig. 22. 6, l. 6 D. de re iud. $42,1,1.18 \S 3 \mathrm{D}$. de dolo 4.3 (und die hierzu gehörige 
Glosse des Accursius), 1. 10 D. de hered. vel. a. vend. 18. 4, 1. 11 eod., 1. $25 \mathrm{D}$. de rei vind. 6. 1, 1. 7 D. de dolo 4. 3, 1. 13 § 4 D. de act. emt. 19.1 , d. 18 \& 2 D. de dolo 4 . $3,1.16$ \& 2 D. de min. 4. 4. Auch werden die ganzen Titel de aed. ed. und de in diem addictione sowohl der Digesten wie des Codex; gleich eingangs als Quellen zitiert. Hervorgehoben sei hier, daß das Promptuarium im Anschlusse an 1. 2 Cod. de resc. vend. 4. 44 den Standpunkt vertritt, es beziehen sich die Bestimmungen des römischen Rechtes betreffs der laesio enormis nicht bloß auf den Verkäufer, sondern auch auf den Käufer: Credo quod Accursius male loquitur in hoc, quod ponit differentiam inter emtorem et venditorem . . . . ideo dico, quod eodem modo tenetur emptor sicut venditor, secundum Henr. Bohit, wobei als ein Argument auf 1. 3 \$ 1 D. si mensor fals. mod. dixerit 11. 6 und einige andere Stellen verwiesen wird. (Über diese nach römischem Recht gewiß unzutreffende Ansicht siehe Zachariä v. Lingenthal in der Sav.-Z. Bd. IV R. A. S. $58 \mathrm{ff}$. und Pfaff, thoer den rechtl. Schutz der wirtsch. Schwächeren S. 64.)

Der Artikel venditio zeigt trotz zahlreicher romanistischer Quellenzitate und der Anführung einer Reihe von romanistischen Schriftstellern wie Bulgarus, Azo, Jason u. a.m. die der römischen Auffassung entgegengesetzte kanonistische Ansicht schon in den ersten Worten, wo es heißt: vendere aliquid carius, quam emptum fuit, si ista intentione emptum fuit, est illicitum.

Im Artikel emphyteuse wird wiederholt das römische Recht als Beleg der vorgetragenen Ansicht herangezogen; insbesondere wird hier bei der Frage emphyteuta an possit meliorationes, accessiones et augmenta impune sine voluntate domini in alium transferre der romanistische Standpunkt, den 1.3 pr. C. de jur. emphyt. 4, 66 enthält, vertreten.

Der Artikel fidejussor zieht gleichfalls römisches Recht sehr häufig heran; in dem dem Dekretalenrecht gewidmeten Abschnitte polemisiert das Promptuarium gegen eine von Jacobus Radewicz ausgesprochene Ansicht und stützt die gegensätzliche Behauptung auf den Gesichtspunkt des arbitrium boni viri und insbesondere auf 1.1 in fine $D$. de jure delib. 28. 8. Ulricus nimmt dabei an, die Frage sei 
auch bei Accursius und Jason de Mayno in gleichem Sinne entschieden : et ita credo Accursius et Ja. de Mayno sensisse.

Der Artikel fraus beruht im wesentlichen auf den römischen Quellen. Ulricus erwähnt, daß zwei Auffassungen bestanden haben; nach der einen sei das in fr. legis agere auf dreifache, nach der anderen auf vierfache Weise möglich. Übrigens verweist er auf den Artikel lex sub f. (quibus et quot modis traus fit legi R. lex f.). Dort wird (Bd. II Blatt 11) mit den Beispielen der Glosse die Auffassung, es erfolge die fraus legi: de re ad rem, de persona ad personam, de uno contractu ad alium und de uno contractu ad eundem contractum vertreten: Bezüglich der Frage, ob im fraudare überhaupt ein sacrilegium gelegen sei, wird auf den diesbezüglichen Artikel hingewiesen.

Im Artikel infa mia wird römisches Recht häufig zitiert. Gestützt auf eine Reihe von Digestenstellen will Ulricus hier den von ihm aus der Literatur mitgeteilten Auffassungen nicht beipflichten und dem Richter eine größere Freiheit gewähren: quia in hac materia officium iudicis latissime patet.

Im Artikel interdictum wird ausschließlich der kirchenrechtliche Begriff erörtert.

Im Artikel iudex, iudicium, ius wird das römische Recht eingehend berücksichtigt; Digesten und Codexstellen sind in reicher Anzahl zitiert, bald aus erster, bald aus zweiter Hand; besonders viel romanistische Quellen sind dabei in dem Abschnitte, der sich mit dem Rechte der Clementinen beschäftigt, angeführt. Gelegentlich tritt - neben der Anführung fremder Ansichten, deren Autoren immer nominatim bezeichnet werden - Ulricus mit eigenen Ansichten hervor, die allerdings größtenteils sich in Polemiken gegenüber zitierten Autoritäten äußern (z. B. Blatt 429 verso ego credo, nos non credimus u. a. m. Auch wird hier reichliche Kasuistik betrieben und immer wieder die Frage aufgeworfen, wie dieser oder jener Fall zu entscheiden sei. So bespricht auf Blatt 444 Ulricus die Frage, welche Mittel den Freunden eines zum Tode Verurteilten zu Gebote stehen gegenüber dem Umstand, daß auf seiten des Richters inimicitia vorgelegen sei. 
In dem Artikel mandatum zitiert das Promptuarium zahlreiche Digestenstellen. Daß die Darstellung der Lehre nicht von romanistischer Auffassung ausgeht, zeigt wohl schon der Satz: mandati fines non excedit, qui facit id, quod non multum differt a mandato, der wohl mit dem in 1. 5 \$2 D. mand. 17. 1 ausgesprochenen Prinzip nicht im Einklange steht.

Im Artikel mora wird am eingehendsten die Frage nach der purgatio morae erörtert. Trotz der relativen Kürze des Artikels - er umfaßt bloß 5 Spalten - sind hier eine Menge von Digesten- und Codexstellen als Beleg der vertretenen Ansichten herangezogen.

Interessant ist der Artikel moneta, in dem zuvörderst die Frage aufgeworfen wird: moneta si facta est vilior, an debitor liberetur solvendo eandem. Römische Quellenstellen bilden auch hier vielfach die Grundlage für die aufgeworfenen Fragen, wie bei der folgenden: monetae duae si currunt in eodem loco, an de utraque possit fieri solutio u. a. m.

Im Artikel mutuum finden wir dagegen - soviel ich zu sehen vermochte - auffallenderweise keinerlei Hinweise auf die römischen Quellen.

Daß der Artikel novatio im ganzen bloß 7 Zeilen umfaßt, sei der Kuriosität halber hier angeführt.

Der Artikel peculium beschränkt sich, wie so manche anderen Teile des Werkes, auf einen Teil des corpus jur. canon. Hier auf den liber Sextus. Die Terminologie ist, wie in den meisten juristisch relevanten Abschnitten, die der römischen Quellen. - Erörtert wird unter anderem auch die Frage, ob, wenn ein advocatus, notarius, tabellio als filii familias etwas erwarben, dies als peculium castrense anzusehen sei oder nicht.

Über das postliminium handeln nur wenige Zeilen; die Auseinandersetzungen erfolgen unter Berufung auf einige römische Quellenstellen, wobei hervorgehoben wird, daß, im Gegensatz zum römischen Recht, nach dem jus canonicum nicht in allen Fällen die rescissoria gewährt wird.

Die Darstellung der Lehre von der praescriptio ist. reich an zur Begründung der aufgestellten Meinungen herangezogenen römischen Quellenstellen. Wie in sehr vielen 
Das Promptuar. jur. d. Reichskanzlers u. Bisch. Ulr. v. Albeck. 171

anderen Artikeln ist auch hier die Kasuistik stark vorwaltend. Die Auffassung, daß die praescriptio auf dem jus naturale beruhe, wird bekämpft; als Grund derselben wird angegeben: praescriptio contra negligentes est inducta; praescriptio est introducta in odium negligentiarum res suas petentium et recipitur bona fides et iustus titulus. Bonafides debet esse continuata de iure canonico, licet secus de iure civili. Mit der Lehre von der praescriptio wird natürlich auch die der usucapio verknüpft und bemerkt: mala fides superveniens interrumpit usucapionem.

Im Artikel prodigus wird eine kurze, im wesentlichen mit dem ausgebildeten römischen Recht übereinstimmende Darstellung gegeben.

Der Artikel Quarta erörtert Fragen aus der Lehre von der Quarta Falcidia und Trebellianica. Dabei wird dem Kanonisten besonders scharf eingeschärft: et nota, tu canoniste, quod Trebelliana de toto fideicommisso detrahitur, Falcidia vero particulariter de singulis legatis.

Die Artikel solutio und stipulatio sind rein referierend abgehalten; im letzteren äußert der Vf., unter Berufung auf eine Digestenstelle, seine von der kanonistischen Lehre abweichende Anschauung.

Der Artikel tutela unterscheidet zwischen testamentarischer, gesetzlicher und dativer Tutel; verschiedene Fragen werden aufgeworfen, ohne an dieser Stelle erledigt zu werden. Diesbezüglich sind Verweisungen auf andere Punkte des Werkes gegeben.

Ein zweites juristisches Werk Albecks findet sich handschriftlich sub Fol. 623 (alte Seckauer Nummer 83) gleichfalls auf der Grazer Universitätsbibliothek. Es ist ein Quartband von 496 Seiten, jede Seite 40-45 Zeilen enthaltend; die Seiten sind nicht paginiert, wie dies beim Promptuarium der Fall ist. Leider ist die Handschrift nicht vollständig und war sie dies schon nicht, als sie im 17. Jahrhundert ihren jetzigen Einband erhielt. Das lehrt der Augenschein. Auf einem ausgerissenen ersten Blatte wird sie genannt: Summa Ulrici Episcopi Seccoviensis (welche Worte nicht von der Hand des Schreibers der Handschrift herrühren), und der Zettelkatalog der Grazer Bibliothek ver- 
zeichnet sie als summa quaestionum et conclusionum juris ex auctoribus collecta ordine alphabetico. In Schlagworten werden - alphabetisch geordnet - zum größten Teil dieselben Themata abgehandelt wie im Promptuarium, jedoch bloß in größter Kürze. Soweit ich verglichen habe, sind in dieser Arbeit dje wichtigsten Sätze aus dem Promptuarium, meist wörtlich übereinstimmend, übernommen. Die einzelnen im Promptuarium aufgeworfenen Fragen werden mit denselben Worten erwähnt, nicht immer in derselben Reihenfolge abgehandelt, viele weggelassen; auch fehlen hier alle Ausführungen über die Literatur, die Begründung eigener Aufstellungen sowie die im Promptuarium gebotene reiche Kasuistik. Das äußere Ausmaß der einzelnen Artikel in dieser Summa, im Verhältnis zum Promptuarium, möge dies veranschaulichen. Während z. B. die Ausführungen über dolus im Promptuarium 4 Spalten umfassen, hat der Artikel in der Summa bloß 24 Zeilen; der Artikel dominium hat im Promptuarium 13 Spalten, hier bloß 2//2 Seiten. Und ähnlich verhält es sich auch bei den übrigen Artikeln, wie fidejussor 10 Spalten gegenüber 26 Zeilen, infamia (8 Spalten gegenüber 1 Seite), mandatum (11 Spalten gegenüber $1 \frac{1}{2}$ Seiten) usw. ${ }^{1}$ )

Beim Buchstaben $\mathbf{P}$ (mit dem Artikel pena) ist eine gewaltige Lücke in der Handschrift, die sich bis zum Buchstaben $V$ erstreckt; beim Artikel vir setzt das Werk sich fort; am Schlusse fehlen weiters sämtliche Artikel des.Buchstabens $\mathrm{Z}$.

Handelte es sich hier dem Vf. meist um eine kurze Darstellung der einschlägigen Materien, welche im Promptuarium ausführlich behandelt waren, so sind andererseits auch eine Reihe von Artikeln des Promptuars weggelassen und manche andere vorhanden, welche im Promptuarium fehlen. ${ }^{2}$ )

1) Summa bedeutet im 12. Jahrhundert und auch später: Zusammenfassende Darstellung, Nachschlagewerk (Grabmann, Gesch. der scholastischen Methode II 21, 23). Auch handelt es sich bei den Summen meist um zusammenfassende Darstellung verschiedener Disziplinen (Grabmann, Einführung in die Summa theolog. des heil. Thomas (1919) S. 3. Im Sinne eines Nachschlagewerkes ist der Titel für diese Arbeit Albecks gewiß zulässig.

$\left.{ }^{2}\right)$ Ich zähle im ganzen 139 Artikel, die ausschließlich im Promptua- 
Auch sind die exzerpierten Autoren nicht durchwegs dieselben wie im Promptuarium; denn für die Summa kamen Albeck neben den im Promptuar benutzten auch noch Michaelis de Furno ${ }^{1}$ ), Bonaventura ${ }^{2}$ ) und Wilhelm Durantis Speculum in Betracht. Letzterer ist besonders ausgiebig exzerpiert, so daß bei manchen Artikeln der dem Recht des speculum gewidmete Teil den größten Raum in Anspruch nimmt, so z. B. bei den Artikeln dolus, dominium, dos, emphyteuta, mutuum, novatio u. a. m.

In seiner Vorrede äußert sich Ulricus Albeck folgendermaßen: In nomine sanctae et individuae trinitatis. Amen. Quia omnia habere magis vel potius est divinitatis quam humanitatis, ut dicit 1. C. de vet. jur. enuc. . . . . et quia memoria labilis et indiget adiutorio dirigente, idcirco ego Ulricus ep. Secc. iuris canonici doctor minimus, dum libros subscriptos perlegi videlicet quaestiones Michaelis de Furno super tres libros sententiarum et Bonaventurae super quartum; decretum cum omni eius apparatu, Distinctiones Heinrici Bohit in decretales, Lecturas Petri de Ancharano super sextum, Dm. Francisci de Zabarellis, qui postea factus fuit Cardinalis, super Clementinas, speculum Wilhelmi Durantis iudiciale, lecturam Baldi de Perusio super usus feudorum, necnon decisiones rotae, eas sub brevitate discurrendo per titulos quaestionum et conclusionem ad memoriale seu repertorium non sine labore gravi recollegi ... Der Vf. wollte also hier ein Repertorium über die von ihn gelesenen Autoren bieten und erörtert die einzelnen Fragen nach den sententiae, dem Rechte des decretum, den decretales, dem rium, und 237, die ausschließlich in der Summa vorkommen. Die für den Juristen relevanten Artikel finden sich fast durchwegs in beiden Werken.

1) Michaelis de Furno (auch Michael de Lille, de Insulis genannt) schrieb Postillen super cantica, super Lucam et Ioannem u. a. m. Er war doctor Parisiensis. S. über ihn Feret, La faculté de Theologie de Paris III 436; Hurter, Nomenclator literarum II ${ }^{3}$ (1906) 568. Er war Dominikaner; bei Ehrle, Sitzungsberichte der Bayr. Akademie, Phil.hist. Klasse, Jahrg. 1919, S. 34 wird er dagegen als aus dem Weltklerus stammend angeführt.

$\left.{ }^{2}\right)$ Bonaventura, geb. 1221, gest. 1274. Sein bedeutendstes Werk ist sein Kommentar zu den Sentenzen des Lombardus, den Ulricus zitiert; vgl. Buchberger, Kirchl. Handlexikon II $1442 \mathrm{ff}$. und Schulte a. a. O. II 26; ferner Wetzer und Welte, Kirchenlexikon IV $1017 \mathrm{ff}$. 
liber sextus, den Clementinen, nach dem speculum Durantis, dem usus feudorum und den decisiones rotae, wobei die sententiae immer an die Spitze gestellt werden.

Über die Abfassungszeit besagt das Vorwort nichts; doch möchte ich es für wahrscheinlich halten, daß das Promptuarium dem hier erwähnten Werke zeitlich voranging. Während er beim Promptuarium ausdrücklich hervorhebt, daß er es schon als Bischof von Verden in Angriff genommen, führt er hier bloß seine Qualität als episcopus Seccoviensis an; und auch das recollegi kann allenfalls dafür herangezogen werden. Ein weiterer Anhaltspunkt ließe sich vielleicht auch aus den Worten, welche er dem Namen des Franciscus de Zabarellis beifügt: qui postea factus fuit Cardinalis, gewinnen.

Mag Albecks Promptuarium - denn dieses kommt vor allem in Betracht - vom Standpunkte des Kanonisten aus größeren oder geringeren Wert besitzen, eines ist wohl sicher, daß ein Hinweis auf diese Arbeit, als eines der nicht zahlreichen Produkte populärer Literatur aus Österreich, immerhin am Platze sein dürfte. (Über die populäre Literatur in Österreich s. Luschin, Handbuch der österr. Reichsgeschichte I ${ }^{2}$ S. 143 und Hasenöhrl, Beiträge zur Gesch. der Rechtsbildung und der Rechtsquellen im Archiv für österr. Gesch. Bd. 93 S. 304 ff., Separatabdruck S. 57 ff.)

Albecks schriftstellerische Tätigkeit ist, soweit ich in der Literatur zu sehen vermag, gänzlich unbekannt. Der ungetwöhnlich große Umfang des Promptuariums sowie der bald nach Vollendung desselben erfolgte Tod des Verfassers mögen hierzu beigetragen haben. Daß im Praesulatus Seccoviensis ihrer freundlich gedacht wird, ist selbstverständlich. Dortselbst heißt es p. 524: De anno praefato bibliotheca nostra insignes commentarios in leges canonicas ab Udalrico episcopo seccoviensi confectos retinet, cum hac inscriptione: Udalricus episcopus Seccov. Prov. Salzburg. anno D. 1408, dum adhuc ecclesiae praeesset Verdensi in provincia Moguntina, conscripsit promptuarium juris secundum alphabetum, quod emulgavit anno 1422 in crastino conceptionis virginis gloriosae Pontif. Papae Martini anno VI regnante Sigis. Romanorum et Ungariae et Bohemiae 
Rege. Abgesehen davon, finde ich in der Literatur nur bei Klein, Gesch. des Christentums in Österr, und Steiermark III (1841) S. 397 die Bemerkung: „Er war Doctor des Kirchenrechts und verfaßte mehrere Schriften, von denen aber nur zwei dem Titel nach bekannt sind, nämlich ein Lexikon, in welchem Gegenstände des Kirchenrechtes in alphabetischer Ordnung beschrieben waren, und eine Erklärung der Kirchengesetze." (Wörtlich gleichlautend bei Ebeling, Die deutschen Bischöfe II S. 439.) Auch in Hurters sorgfältiger Arbeit Nomenclator literarius fehlt der Name Albecks, ein Zeichen, daß seine Arbeiten einem weiteren Kreise unbekannt geblieben sind. $\mathrm{Ab}$ gesehen von den beiden Handschriften der hiesigen Universitätsbibliothek finde ich bei Haenel, Catalog. libr. manuscript. p. 458 ein Memoriale juris angeführt, das sich auf der Straßburger Bibliothek befindet, sowie im Catalog. cod. Latinarum der Münchener Bibliothek Tom. II pars 2 p. 61 sub Nr. 592 die Notiz: Ulrici de Albeck canonici Augustani Spirensis et Basilensis Repertorium super commentarios Henr. Bouhic in libros decretalium 12254 Raitenb. 54 in $2^{\circ}$ XV 88 fol. Danach ist wohl das Zitat bei Schulte a. a. O. II 268 ungenau, wo diese Arbeit einem Heinricus de Albeck j. c. Doctor Augusteus, Spirensis zugeschrieben wird. Schulte entnahm das Zitat dem Handschriftenkatalog der Universitätsbibliothek Erlangen von Irmischer. - Soweit ich der Frage nach Schriften Albecks nachgehen konnte, fand ich in keinem der gedruckten Verzeichnisse von Handschriften Werke von Albeck angeführt. Kollege Wolkan hatte die Güte, mir mitzuteilen, daß in keiner der ihm bekannten Handschriftensammlungen sich ein weiteres Manuskript von Albeck vorfinde. - Über den Lebenslauf Ulrich $\nabla$. Albecks, insbesondere über seinen Studiengang, seine kirchliche Tätigkeit als Bischof von Verden an der Aller und von Seckau, seine Wirksamkeit als Bevollmächtigter und Kanzler Ruprechts von der Pfalz und seine Teilnahme an den Konzilien zu Pisa und Konstanz, dem Fürstentag zu Marburg, der Salzburger Synode von 1418 hoffe ich an anderer Stelle demnächst berichten zu können. 